\title{
Review Article \\ The ECM-Cell Interaction of Cartilage Extracellular Matrix on Chondrocytes
}

\author{
Yue Gao, Shuyun Liu, Jingxiang Huang, Weimin Guo, Jifeng Chen, Li Zhang, Bin Zhao, \\ Jiang Peng, Aiyuan Wang, Yu Wang, Wenjing Xu, Shibi Lu, Mei Yuan, and Quanyi Guo
}

Institute of Orthopedics, Chinese PLA General Hospital, Beijing 100853, China

Correspondence should be addressed to Quanyi Guo; guoquanyi301@gmail.com

Received 14 February 2014; Accepted 24 April 2014; Published 18 May 2014

Academic Editor: Albana Ndreu-Halili

Copyright (C) 2014 Yue Gao et al. This is an open access article distributed under the Creative Commons Attribution License, which permits unrestricted use, distribution, and reproduction in any medium, provided the original work is properly cited.

Cartilage extracellular matrix (ECM) is composed primarily of the network type II collagen (COLII) and an interlocking mesh of fibrous proteins and proteoglycans (PGs), hyaluronic acid (HA), and chondroitin sulfate (CS). Articular cartilage ECM plays a crucial role in regulating chondrocyte metabolism and functions, such as organized cytoskeleton through integrinmediated signaling via cell-matrix interaction. Cell signaling through integrins regulates several chondrocyte functions, including differentiation, metabolism, matrix remodeling, responses to mechanical stimulation, and cell survival. The major signaling pathways that regulate chondrogenesis have been identified as wnt signal, nitric oxide (NO) signal, protein kinase C (PKC), and retinoic acid (RA) signal. Integrins are a large family of molecules that are central regulators in multicellular biology. They orchestrate cell-cell and cell-matrix adhesive interactions from embryonic development to mature tissue function. In this review, we emphasize the signaling molecule effect and the biomechanics effect of cartilage ECM on chondrogenesis.

\section{What Is ECM?}

In biology, the extracellular matrix (ECM) is the extracellular part of multicellular structure (e.g., organisms, tissues, and biofilms) that typically provides structural and biochemical support to the surrounding cells [1]. Because multicellularity evolved independently in different multicellular lineages, the composition of ECM varies between multicellular structures; however, cell adhesion, cell-to-cell communication, and differentiation are common functions of the ECM [2].

Cartilage ECM is composed primarily of COLII and large networks of PGs that contain GAG such as HA and CS. Because cartilage shows little tendency for self-repair, injuries remain unhealed for years and can lead to further degeneration [3]. Cartilaginous ECM is remodeled continuously by a combination of production, degradation by matrix metalloproteinases (MMPs), and inhibition of MMPs activity by tissue inhibitors of MMPs [4]. The ECM of articular cartilage is a unique environment. ECM components through their action on integrin clustering are involved in cell adhesion, cortical actin cytoskeleton organization, and cell spreading [5].
The ECM is composed of large proteoglycans (PGs) that contain glycosaminoglycan (GAG), hyaluronic acid (HA), fibers, and other molecular components about fibronectin and laminin. Fibers contain elastin and collagen that include fibrillar (types I, II, III, V, and XI), FACIT (types IX, XII, and XIV), short chain (types VIII and X), basement membrane (type IV), and others (types VI, VII, and XIII) [6] (Figure 1). In the ECM, especially the basement membrane, the multidomain proteins perlecan, agrin, and COLXVIII are the main proteins to which heparan sulfate attaches [7]. At last, there are important molecular components called integrins. Integrins are transmembrane receptors that mediate the attachment between a cell and its surroundings, such as other cells or the extracellular matrix (ECM). In signal transduction, integrins pass information about the chemical composition and mechanical status of the ECM into the cell. Therefore, in addition to transmitting mechanical forces across otherwise vulnerable membranes, they are involved in cell signaling and the regulation of cell cycle, shape, and motility. 


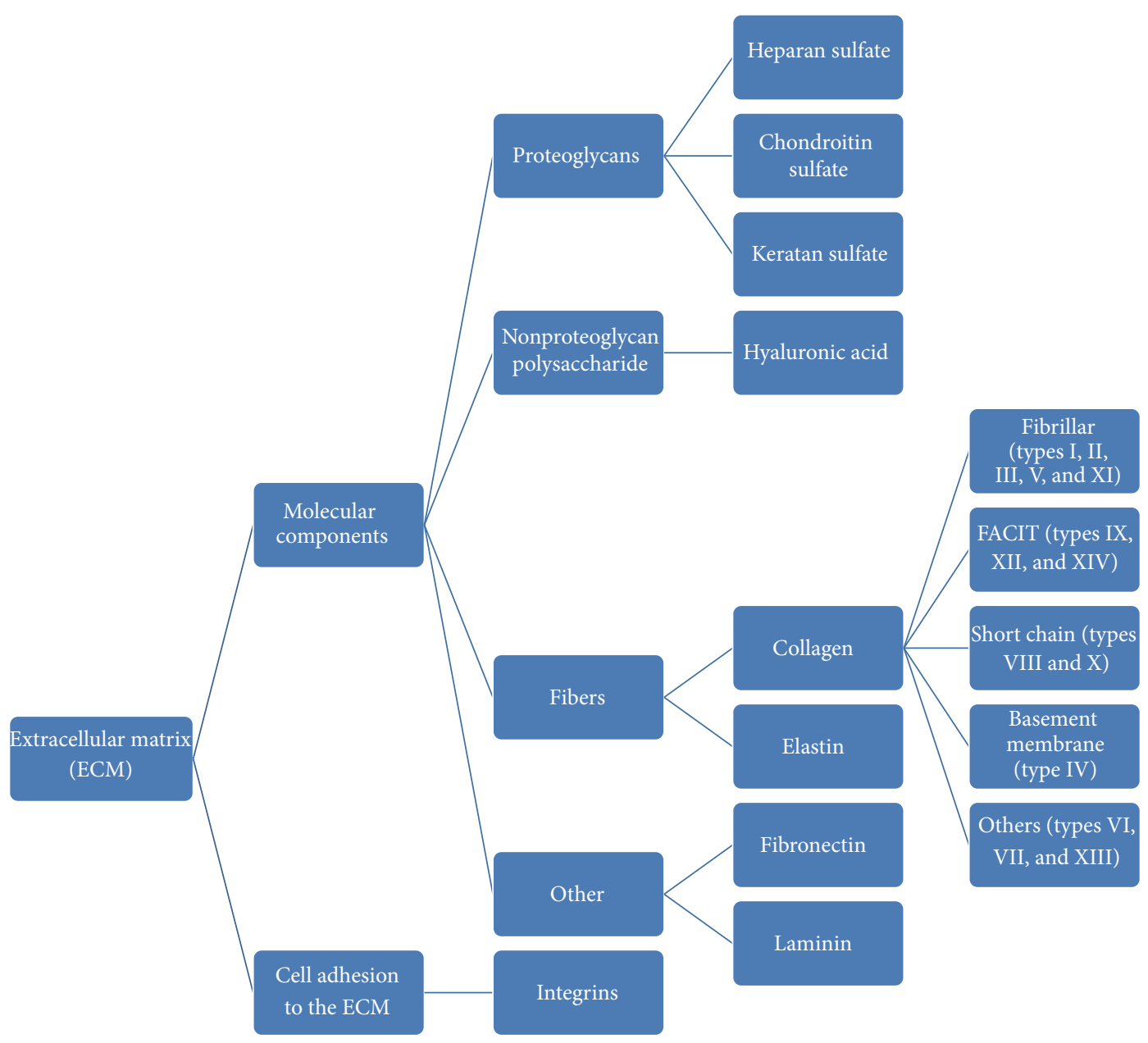

FiguRe 1

\section{What Is the Function of ECM?}

Because of its diverse nature and composition, the ECM can have many functions, such as providing support, segregating tissues, and regulating intercellular communication. The ECM regulates a cell's dynamic behavior. In addition, it stores a wide range of cellular growth factors and acts as a local depot for them. Changes in physiological conditions can trigger protease activities that cause the local release of such depots. This situation allows for the rapid and local growth-factor-mediated activation of cellular functions. The formation of the ECM is essential for processes such as growth, wound healing, and fibrosis. PGs have a net negative charge that attracts positively charged sodium ions which attracts water molecules via osmosis. PGs can keep the ECM and resident cells hydrated. PGs may also help trap and store growth factors within the ECM. Once secreted, the molecules aggregate with the existing matrix. Resident cells intracellularly produce the components of the ECM via exocytosis [6].

Articular cartilage ECM plays a crucial role in regulating chondrocyte functions via cell-matrix interaction, organized cytoskeleton, and integrin-mediated signaling. The ECM has a significant effect on the swelling behavior and osmotic environment of chondrocytes [8]. Factors produced by chondrocytes can affect the synthesis of the ECM. These factors are ILs, basic fibroblast growth factor (BFGF), bone morphogenic proteins (BMPs), and insulin-like growth factor (IGF). Cell signaling mediated by integrin regulates several chondrocyte functions, including differentiation, matrix remodeling, responses to mechanical stimulation, and cell survival [9-11]. CS and HA influence the proliferation and differentiation of chondrocytes. Scaffolds composed of COLII, CS, and HA may create an environment that can preserve the normal phenotype of cells to promote regeneration of cartilage-like constructs [12]. CS contributes to the tensile strength of cartilage, tendons, ligaments, and walls of the aorta. Low-molecular-weight isoforms of the aggrecanases are responsible for the cytokine-induced proteolysis of aggrecan in a porcine chondrocyte culture system [13, 14]. Abnormal contact between chondrocytes and the ECM has serious consequences. Chondrocytes isolated from Racldeficient growth plates show reduced adhesion to COLII and fibronectin [15]. To provide a larger space to allow for cell proliferation and generation of new ECM, we found that a COLII scaffold composed of collagen with genipin is similar to natural ECM; the application of CS can increase mRNA and DNA biosynthesis and promote cell metabolism the 
same as with the acid mucopolysaccharide HA, with strong bonding and hydrophilic properties, to retain moisture, so that it better resembles the natural ECM and promotes cell proliferation [12].

Interactions between chondrocytes and the ECM regulate many biological processes important to homeostasis and repair of articular cartilage, including cell attachment, growth, differentiation, and survival. Integrins have two main functions: (1) attachment of the cell to the ECM and (2) signal transduction from the ECM to the cell.

However, they are also involved in a wide range of other biological activities, including immune patrolling, cell migration, and binding to cells by certain viruses, such as adenovirus, echovirus, hantavirus, and foot and mouth disease viruses.

A prominent function of the integrins is seen in the molecule GPIIbIIIa, an integrin on the surface of blood platelets (thrombocytes) responsible for attachment to fibrin within a developing blood clot. This molecule dramatically increases its binding affinity for fibrin/fibrinogen through association of platelets with exposed collagens in the wound site. Upon association of platelets with collagen, GPIIbIIIa changes shape, allowing it to bind to fibrin and other blood components to form the clot matrix and stop blood loss. Integrins are adhesion receptor heterodimers that transmit information from the ECM to the cell through activation of cell signaling pathways. The integrins are a large family of heterodimeric cell adhesion receptors involved in cell-cell and cell-matrix interactions [16, 17].

\section{ECM and Cell Interaction}

The general concept of chondrogenesis is as follows. In embryogenesis, the skeletal system is derived from the mesoderm germ layer. Chondrogenesis is the process by which cartilage is formed from condensed mesenchyme tissue, which differentiates into chondrocytes and begins secreting the molecules that form the ECM.

The relationship between ECM and chondrogenesis should be discussed here. The 3D environment of the ECM guides the morphogenesis of tissue types with anisotropic structures [18]. Chondrocyte differentiation is a multistep process characterized by successive changes in cell morphologic features and gene expression. Early in fetal development, the greater part of the skeleton is cartilaginous. This temporary cartilage is gradually replaced by bone (endochondral ossification), a process that ends at puberty. In contrast, the cartilage in the joints remains unossified during the whole life and is, therefore, permanent. During the early phase of the chondrocyte life cycle, cell-cell adhesion occurs via molecules such as $\mathrm{N}$-cadherin. At later stages, such as in growth-plate chondrocytes, adhesion signaling occurs from ECM proteins via integrin and other ECM receptors. Cellmatrix interactions are also important for chondrogenesis.

3.1. Signals and Integrins. The major signaling pathways that regulate chondrogenesis must play an important role through the cell-matrix interaction. These molecules are bound to plasma membrane or intracellular receptors and are interpreted by complex molecular pathways that use specific combinations of a cell or tissue-specific signaling toolkit, and, by eventually converging on transcription factors, they induce changes in gene expression. These signals are required to adjust the cellular metabolism to the needs of the tissue and/or organism or to affect the fate of cells: proliferation, differentiation, or apotosis [19] through wnt signal, nitric oxide signal, retinoic acid (RA) signal, and protein kinase $\mathrm{C}$ (PKC).

Wnt9a was shown to be required for chondrocyte proliferation and mediolateral intercalation, cellular mechanisms that mediate extension during zebrafish palate morphogenesis [20]. Frzb and $f z d 7 a$ are dispensable for directed migration of the bilateral trabeculae, but necessary for the convergence and extension of the palatal elements, where the extension process is mediated by chondrocyte proliferation, morphologic change, and intercalation. Bapx1 was specifically downregulated in the wnt $9 a / f r z b / f z d 7 a$ morphants. Overexpression of bapxl can partially rescue the lower jaw elements in $w n t 9 a, f r z b$, and $f z d 7 a$ morphants [21].

Nitric oxide (NO) was recognized as an important second messenger signaling molecule generated from metabolism of L-arginine by the nitric oxide synthase (NOS) family of enzymes [22]. Nitric oxide synthase inhibitor 1-(2[trifluoromethyl] phenyl) imidazole (TRIM) can disrupt chondrogenic differentiation. So TRIM-treated embryo only formed scattered chondrocyte clusters. TRIM treatment could be reasoned by several developmental events, such as failure in identity specification within changes in cell proliferation and survival, and/or defects in chondrogenic differentiation. NO might function upstream of histone acetylation and/or through nonacetylation pathways (e.g., through S-nitrosylation; or NO may directly target the expression of chondrogenic genes). TRIM inhibited chondrogenic differentiation, which were mediated through impaired nitric oxide (NO) production without appreciable effect on global protein S-nitrosylation. TRIM perturbed Hox gene patterning and caused histone hypoacetylation [23]. NO regulates cartilage degradation by causing dedifferentiation and apoptosis of chondrocytes via activation of ERK1/2 and p38 [24].

RA is responsible for most of the activity of vitamin $A$ and saves visual pigment effects that require retinal (retinaldehyde) and cell metabolism effects that may require retinol itself. Also, some biochemical functions necessary for fertility in vitamin A deficient male and female mammals originally appeared to require retinol for rescue, but this is due to a requirement for local conversion of retinol to RA, as administered RA does not reach some critical tissues unless given in high amounts. RA significantly increased the motility of neural crest cells, as shown by the woundhealing assay, and inhibited their proliferation. Cartilage elements originate from midbrain neural crest cells. RA can cause abnormal craniofacial cartilage development in other vertebrates, resulting in dose- and stage-dependent losses of $d l x$ homeobox gene expression in several regions of the embryo [25, 26]. 
During chondrogenesis, reversible phosphorylation of key target proteins is of particular importance during this process. Among protein kinases known to be involved in these pathways, PKC subtypes play pivotal roles. PKC is a quintessential regulator of chondrogenesis. PKCs regulate the chondrocyte phenotype via the actin cytoskeleton. PKC exerts its chondrogenesis-promoting effect via the ERKMAPK pathway. PKC mediates chondrogenesis via the ERK1/2 pathway. Chondrocyte de- and redifferentiation are regulated by PKC and MAPK signaling. PKC mediates the effects of IGF-1 and EGF during chondrogenesis. PKCdependent regulation of chondrogenesis is via cell adhesion molecules [19].

In all, 24 unique integrin dimers are formed in vivo from the $18 \alpha$ and $8 \beta$ subunits found in mammalian cells. The composition of the ECM is expressed in a given cell type. Integrins and cell signals can regulate cell shape and affinity. Chondrocytes express a subset of integrin subunits including fibronectin receptors, a laminin receptor, and collagen receptors [10, 27-32]. The $\beta 1$ chain is a component of most chondrocyte integrins. Cartilage-specific deactivation of the $\beta 1$-integrin gene results in severe changes in the cartilage phenotype [33]. Chondrocytes from knock-out mice show abnormal cell shape, reduced proliferation, and deregulated expression of cell-cycle proteins, including D-type cyclins and cyclin-dependent kinase inhibitors. In vitro experiments also suggest that the loss of $\beta 1$ and $\alpha \mathrm{n} \beta 5$ integrin promotes apoptosis in growth-plate chondrocytes and that antibodies against $\beta 1, \alpha 2$, or $\alpha 3$ integrin [33-35] repress hypertrophic differentiation and decrease chondrocyte survival.

Integrin-mediated activation of members of the mitogenactivated protein kinase family plays a key role in transmitting signals regulating chondrocyte gene expression. Some research has verified with isotope-labeled monoclonal antibodies that chondrocyte phenotype remains may be due to the attachment mediated via integrin, including members of both the $\beta 1$ and $\beta 3$ subunit families. Then, chondrocytes showed significant attachment to fibronectin matrix Gla protein, osteopontin, bone sialoprotein II, vitronectin, and COLII and VI [36, 37], which suggests a link between matrix synthesis and integrin expression in chondrocytes. Chondrocytes express several members of the integrin family, including $\alpha 5 \beta 1$, the primary chondrocyte receptor for fibronectin. The $\alpha 5 \beta 1$ integrin provides matrix survival signals for normal and osteoarthritic human articular chondrocytes, to prevent apoptosis. Therefore, $\beta$-integrin-mediated chondrocyteECM interactions are decreased in osteoarthritic cartilage, which suggests that perturbations of chondrocyte-matrix signaling occurs during $\mathrm{OA}[10,38,39]$. $\beta 1$ integrin, the protein encoded by the ITGB1 gene (also known as CD29 and VLAB) [17], is a multifunctional protein involved in cell-matrix adhesion, cell signaling, cell adhesion, protein binding, and receptor-mediated activity. The $\beta 1$-integrin family of cellsurface receptors appears to play a major role in mediating cell-matrix interactions that are important in regulating these fundamental processes.

Degradation of HA results in chondrocyte aggregation and then reduces chondrocyte apoptosis. As well, $\beta 1$-integrincollagen interaction reduces chondrocyte apoptosis [40], to achieve their goals by antagonizing hyaluronidase. So, like integrin-deficient chondrocytes, adhesion to the ECM decreased in Flnb (-/-) chondrocytes, and inhibition of $\beta 1$ integrin in these cells further impaired cell spreading [41]. TGF- $\beta 1$ and integrin stimuli interact before Smad 2 and -3 phosphorylation in the cytoplasm of chondrocytes, which regulates the expression of ECM components in chondrocytes. Under culture and seeding conditions, $\beta 1, \alpha 5 \beta 1$, and $\alpha \mathrm{v} \beta 5$ integrins [42] mediate human chondrocyte adhesion to cartilage. These chondrocyte integrins have a potential role in the initial adhesion and retention of chondrocytes at a cartilage defect site. The fibronectin receptor $(\alpha 5 \beta 1$ integrin), in conjunction with its ligand fibronectin, the GPIIb/IIIa receptor and the integrin-linked kinase, integrin cytoplasmic-domain-associated protein 1 , and CD47 pathway play a pivotal role in dedifferentiation of chondrocytes [16]. TGF- $\beta 3$, MMP9, MMP13 [43-46], and vascular endothelial growth factor are key regulators for remodeling cartilage tissues. They coordinate matrix degradation and the recruitment and differentiation of osteoprogenitors. IL-1 receptor antagonist upregulates major components of the cartilage ECM genes, so we can use it to protect the ECM for antiinflammatory and chondroprotective therapy.

3.2. Factors and Enzymes. After disruption of cell-matrix interactions and lack of growth factors, certain cells are selected and channelled through proliferation into the new stable phenotype. Chondrocyte mechanoreceptors may incorporate $\beta 1$-integrins and mechanosensitive ion channels linked with key ECM, cytoskeletal, and signaling proteins to maintain the chondrocyte phenotype, prevent chondrocyte apoptosis, and regulate chondrocyte-specific gene expression $[17,47]$. Tumor necrosis factor $\alpha$ (TNF- $\alpha$ ) and interleukins- $1 \beta$ (IL-1 $\beta$ ) cause the release of the stress-injury-related protein to relieve mechanical damage. TNF and IL-1 or anti-Fas antibody growth-regulated oncogene $\alpha$ in ECM can induce chondrocyte apoptosis. Chondrocyte apoptosis and caspase3 activity are associated $[9,48]$. IGF-1 is known to inhibit the catabolic effects of IL-1 on PG synthesis in cartilage explants and suppresses the degradation of ECM components by reducing matrix metalloproteinase-1 (MMP-1) and MMP8 expression and activity [49].

Apoptosis is programmed cell death. Apoptotic cells take the initiative of cell death (necrosis). A disease such as OA can lead to apoptosis. OA results from the aberrant production of inflammatory mediators (cytokines and chemokines) and effectors (MMPs and reactive oxygen and nitrogen species) by chondrocytes [48]. Cartilage oligomeric matrix protein (COMP) plays an important role in cartilage cell-matrix interactions. COMPs induce the survival of the inhibitor of apoptosis family of proteins to lead to the strong inhibition of chondrocyte apoptosis by blocking the activation of caspase3. The synthesis of COMP is regulated by transforming growth factor (TGF) in these 2 regions of the human articular cartilage [50-52]. COMP mutation has a great impact. Although it is a small molecule in the ECM, its mutation is the major reason for pseudoachondroplasia. COMP specifically locates in some cells of the rough endoplasmic reticulum and 
has toxic effects on chondrocyte precursors, thus hindering the formation of cartilage and bone [53-59]. A certain amount of strontium and COMP can maintain the structural integrity of the cartilage collagen and fibronectin $[52,60]$.

3.3. Biomechanics. In terms of biomechanics, cartilage tissue can remodel its ECM in response to alterations in functional demand. The pericellular matrix (PCM) is a narrow tissue region surrounding chondrocytes in articular cartilage, which together with the enclosed cell(s) has been termed the "chondron" [8]. The PCM is rich in fibronectin, PGs (e.g., aggrecan, HA, and decorin), and collagen (types II, VI, and IX) but, as compared with the ECM, is defined primarily by the presence of COLVI. The mechanical properties of PCM relative to those of the ECM can significantly affect the micromechanical environment of the chondrocyte. Changes in the properties of the PCM with osteoarthritis (OA) may alter the stress-strain and fluid-flow environment of chondrocytes $[8,61,62]$.

OA leads to the degradation of the PCM and then alters the cellular environment of cartilage in terms of macroscopic loading features and material properties of the ECM and the chondron. At the microscale, estimates of Young's modulus of the PCM range from about 24 to $59 \mathrm{kPa}$ by the axisymmetric boundary element method. Therefore, the PCM may have an important role in modulating the mechanical environment of the chondrocyte $[63,64]$. Compressed damage by overloading the integrity of the cartilage ECM may cause cell membrane damage and eventually cell death. TNF and IL-1 cause the release of the stress-injury-related protein to relieve mechanical damage $[65,66]$.

\section{Conclusions}

The survey of the considerable domain of definition, components, and ECM-cell interaction of ECM can indicate the amount of knowledge accumulated and the directions of research and applications. It is evident that we are only at the beginning of understanding the precise role of cellmatrix interaction during chondrogenesis and how they are regulated. There are a lot of unexplored fields in this area; exploiting these novel approaches and applying them to not only healthy but also inflammatory chondrocytes may enable us to halt or even reverse disease progression. Shedding more light on exactly how the ECM and cell interact with each other PCM-mediated control mechanisms would open new perspectives for a better understanding of healthy as well as pathological differentiation processes of chondrocytes and may also lead to the development of new therapeutic approaches.

\section{Perspective}

Regulation of cell shape and signaling from cell-cell and cellECM interactions are vital to the maturation of chondrocytes. Determining signaling pathways and targets downstream of these events will aid in the development of novel strategies for cartilage replacement and new approaches for regenerating cartilage and preventing and treating cartilage disorders. Still, illustrating the mechanisms integrating signals from adhesion receptors with those from growth factor and hormone receptors will contribute to a better understanding of physiologic and pathologic endochondral ossification. Complex approaches to gene mutagenesis in mice combined with advanced genomic, proteomic, and imaging tools will provide a powerful stage for rapid progress in these areas.

\author{
Abbreviations \\ BFGF: Basic fibroblast growth factor \\ BMP: Bone morphogenic proteins \\ COLII: Type II collagen \\ COMP: Cartilage oligomeric matrix protein \\ CS: Chondroitin sulfate \\ ECM: Extracellular matrix \\ GAG: Glycosaminoglycan \\ HA: Hyaluronic acid \\ IGF: Insulin-like growth factor \\ ILs: Interleukins \\ ITGB1: Integrin 1 \\ MMPs: Matrix metalloproteinases \\ $\mathrm{Na}^{+}: \quad$ Sodium ions \\ NO: Nitric oxide \\ PCM: Pericellular matrix \\ PGs: Proteoglycans \\ PKC: Protein kinase C \\ RA: Retinoic acid \\ TNF: Tumor necrosis factor \\ TGF: Transforming growth factor.
}

\section{Conflict of Interests}

The authors declare that there is no conflict of interests regarding the publication of this paper.

\section{Acknowledgments}

This work was funded by the Beijing Metropolis Beijing Nova Program (2011115), the National Natural Science Foundation of China (General Program) (31170946), the National Natural Science Foundation of China (Youth Program) (31100696), the National High Technology Research and Development Program of China (2012AA020502), the People's Liberation Army 12th Five-Year Plan Period (Key Program) (BWS11J025), the National Basic Research Program of China (973 Program) (2012CB518106), the National Natural Science Foundation of China (Key Program) (21134004), and the New Drug Creation of the Special Ministry of Science and Technology.

\section{References}

[1] G. Michel, T. Tonon, D. Scornet, J. M. Cock, and B. Kloareg, "The cell wall polysaccharide metabolism of the brown alga Ectocarpus siliculosus. Insights into the evolution of extracellular matrix polysaccharides in Eukaryotes," New Phytologist, vol. 188, no. 1, pp. 82-97, 2010. 
[2] M. Abedin and N. King, "Diverse evolutionary paths to cell adhesion," Trends in Cell Biology, vol. 20, no. 12, pp. 734-742, 2010.

[3] C.-H. Wu, C.-S. Ko, J.-W. Huang, H.-J. Huang, and I.-M. $\mathrm{Chu}$, "Effects of exogenous glycosaminoglycans on human chondrocytes cultivated on type II collagen scaffolds," Journal of Materials Science: Materials in Medicine, vol. 21, no. 2, pp. 725729, 2010.

[4] I. Takahashi, K. Onodera, J.-W. Bae, H. Mitani, Y. Sasano, and H. Mitani, "Age-related changes in the expression of gelatinase and tissue inhibitor of metalloproteinase genes in mandibular condylar, growth plate, and articular cartilage in rats," Journal of Molecular Histology, vol. 36, no. 5, pp. 355-366, 2005.

[5] S. Honoré, V. Pichard, C. Penel et al., "Outside-in regulation of integrin clustering processes by ECM components per se and their involvement in actin cytoskeleton organization in a colon adenocarcinoma cell line," Histochemistry and Cell Biology, vol. 114, no. 4, pp. 323-335, 2000.

[6] P. G, “The extracellular matrix and cell adhesion," in Cells, B. Lewin, L. Cassimeris, V. Lingappa, and G. Plopper, Eds., 2007.

[7] E. Sweeney, D. Roberts, and O. Jacenko, "Altered matrix at the chondro-osseous junction leads to defects in lymphopoiesis," Annals of the New York Academy of Sciences, vol. 1237, no. 1, pp. 79-87, 2011.

[8] F. Guilak, L. G. Alexopoulos, M. L. Upton et al., "The pericellular matrix as a transducer of biomechanical and biochemical signals in articular cartilage," Annals of the New York Academy of Sciences, vol. 1068, no. 1, pp. 498-512, 2006.

[9] B. A. Fischer, S. Mundle, and A. A. Cole, "Tumor necrosis factor-alpha induced DNA cleavage in human articular chondrocytes may involve multiple endonucleolytic activities during apoptosis," Microscopy Research and Technique, vol. 50, no. 3, pp. 236-242, 2000.

[10] R. F. Loeser, "Integrins and cell signaling in chondrocytes," Biorheology, vol. 39, no. 1-2, pp. 119-124, 2002.

[11] S. Ab-Rahim, L. Selvaratnam, and T. Kamarul, "The effect of TGF- $\beta 1$ and $\beta$-estradiol on glycosaminoglycan and type II collagen distribution in articular chondrocyte cultures," Cell Biology International, vol. 32, no. 7, pp. 841-847, 2008.

[12] C.-S. Ko, J.-P. Huang, C.-W. Huang, and I.-M. Chu, “Type II collagen-chondroitin sulfate-hyaluronan scaffold cross-linked by genipin for cartilage tissue engineering," Journal of Bioscience and Bioengineering, vol. 107, no. 2, pp. 177-182, 2009.

[13] T. K. Hensch, "Critical Period Mechanisms in Developing Visual Cortex," Current Topics in Developmental Biology, vol. 69, pp. 215-237, 2005.

[14] A. J. Powell, C. B. Little, and C. E. Hughes, "Low molecular weight isoforms of the aggrecanases are responsible for the cytokine-induced proteolysis of aggrecan in a porcine chondrocyte culture system," Arthritis and Rheumatism, vol. 56, no. 9, pp. 3010-3019, 2007.

[15] A. Woods, G. Wang, and F. Beier, "Regulation of chondrocyte differentiation by the act in cytoskeleton and adhesive interactions," Journal of Cellular Physiology, vol. 213, no. 1, pp. 1-8, 2007.

[16] U. R. Goessler, P. Bugert, K. Bieback et al., "Differential modulation of integrin expression in chondrocytes during expansion for tissue engineering," In Vivo, vol. 19, no. 3, pp. 501-508, 2005.

[17] M. Shakibaei, C. Csaki, and A. Mobasheri, "Diverse roles of integrin receptors in articular cartilage," Advances in Anatomy, Embryology, and Cell Biology, vol. 197, pp. 1-60, 2008.
[18] B. Lanfer, F. P. Seib, U. Freudenberg et al., "The growth and differentiation of mesenchymal stem and progenitor cells cultured on aligned collagen matrices," Biomaterials, vol. 30, no. 30, pp. 5950-5958, 2009.

[19] C. Matta and A. Mobasheri, "Regulation of chondrogenesis by protein kinase C: emerging new roles in calcium signalling," Cellular Signalling, vol. 26, no. 5, pp. 979-1000, 2014.

[20] M. Dougherty, G. Kamel, M. Grimaldi et al., "Distinct requirements for wnt9a and irf6 in extension and integration mechanisms during zebrafish palate morphogenesis," Development, vol. 140, no. 1, pp. 76-81, 2013.

[21] G. Kamel, T. Hoyos, L. Rochard et al., "Requirement for frzb and fzd7a in cranial neural crest convergence and extension mechanisms during zebrafish palate and jaw morphogenesis," Developmental Biology, vol. 381, no. 2, pp. 423-433, 2013.

[22] S. Moncada and A. Higgs, "The L-arginine-nitric oxide pathway," The New England Journal of Medicine, vol. 329, no. 27, pp. 2002-2012, 1993.

[23] Y. Kong, M. Grimaldi, E. Curtin et al., "Neural crest development and craniofacial morphogenesis is coordinated by nitric oxide and histone acetylation," Chemistry \& Biology, no. 21, pp. 1-14, 2014.

[24] S.-J. Kim, N.-W. Ju, C.-D. Oh et al., "ERK-1/2 and p38 kinase oppositely regulate nitric oxide-induced apoptosis of chondrocytes in association with p53, caspase-3, and differentiation status," Journal of Biological Chemistry, vol. 277, no. 2, pp. 1332$1339,2002$.

[25] D. L. Ellies, R. M. Langille, C. C. Martin, M.-A. Akimenko, and M. Ekker, "Specific craniofacial cartilage dysmorphogenesis coincides with a loss of dlx gene expression in retinoic acidtreated zebrafish embryos," Mechanisms of Development, vol. 61, no. 1, pp. 23-36, 1997.

[26] B. Kinikoglu, Y. Kong, and E. C. Liao, "Characterization of cultured multipotent zebrafish neural crest cells," Experimental Biology and Medicine, vol. 239, no. 2, pp. 159-168, 2013.

[27] M. Shakibaei, B. Zimmermann, and H.-J. Merker, "Changes in integrin expression during chondrogenesis in vitro: an immunomorphological study," Journal of Histochemistry and Cytochemistry, vol. 43, no. 10, pp. 1061-1069, 1995.

[28] R. F. Loeser, "Chondrocyte integrin expression and function," Biorheology, vol. 37, no. 1-2, pp. 109-116, 2000.

[29] A. Van der Flier and A. Sonnenberg, "Function and interactions of integrins," Cell and Tissue Research, vol. 305, no. 3, pp. 285298, 2001.

[30] R. O. Hynes, "Integrins: bidirectional, allosteric signaling machines," Cell, vol. 110, no. 6, pp. 673-687, 2002.

[31] D. G. Stupack and D. A. Cheresh, "Get a ligand, get a life: integrins, signaling and cell survival," Journal of Cell Science, vol. 115, no. 19, pp. 3729-3738, 2002.

[32] M. A. Arnaout, B. Mahalingam, and J.-P. Xiong, "Integrin structure, allostery, and bidirectional signaling," Annual Review of Cell and Developmental Biology, vol. 21, pp. 381-410, 2005.

[33] A. Aszodi, E. B. Hunziker, C. Brakebusch, and R. Fässler, " $\beta 1$ integrins regulate chondrocyte rotation, G1 progression, and cytokinesis," Genes and Development, vol. 17, no. 19, pp. 24652479, 2003.

[34] M. S. Hirsch, L. E. Lunsford, V. Trinkaus-Randall, and K. K. Svoboda, "Chondrocyte survival and differentiation in situ are integrin mediated," Developmental Dynamics, vol. 210, no. 3, pp. 249-263, 1997. 
[35] W. Wang and T. Kirsch, "Annexin V/ $\beta 5$ integrin interactions regulate apoptosis of growth plate chondrocytes," Journal of Biological Chemistry, vol. 281, no. 41, pp. 30848-30856, 2006.

[36] R. F. Loeser, "Integrin-mediated attachment of articular chondrocytes to extracellular matrix proteins," Arthritis and Rheumatism, vol. 36, no. 8, pp. 1103-1110, 1993.

[37] R. F. Loeser, "Modulation of integrin-mediated attachment of chondrocytes to extracellular matrix proteins by cations, retinoic acid, and transforming growth factor $\beta$," Experimental Cell Research, vol. 211, no. 1, pp. 17-23, 1994.

[38] G. Lapadula, F. Iannone, C. Zuccaro et al., "Chondrocyte phenotyping in human osteoarthritis," Clinical Rheumatology, vol. 17, no. 2, pp. 99-104, 1998.

[39] J. I. Pulai, M. Del Carlo Jr., and R. F. Loeser, "The $\alpha 5 \beta 1$ integrin provides matrix survival signals for normal and osteoarthritic human articular chondrocytes in vitro," Arthritis and Rheumatism, vol. 46, no. 6, pp. 1528-1535, 2002.

[40] L. Cao, V. Lee, M. E. Adams et al., “ $\beta 1$-Integrin-collagen interaction reduces chondrocyte apoptosis," Matrix Biology, vol. 18, no. 4, pp. 343-355, 1999.

[41] J. Lu, G. Lian, R. Lenkinski et al., "Filamin B mutations cause chondrocyte defects in skeletal development," Human Molecular Genetics, vol. 16, no. 14, pp. 1661-1675, 2007.

[42] M. S. Kurtis, T. A. Schmidt, W. D. Bugbee, R. F. Loeser, and R. L. Sah, "Integrin-mediated adhesion of human articular chondrocytes to cartilage," Arthritis and Rheumatism, vol. 48, no. 1, pp. 110-118, 2003.

[43] N. Ortega, D. Behonick, D. Stickens, and Z. Werb, "How proteases regulate bone morphogenesis," Annals of the New York Academy of Sciences, vol. 995, pp. 109-116, 2003.

[44] K. H. Park and K. Na, "Effect of growth factors on chondrogenic differentiation of rabbit mesenchymal cells embedded in injectable hydrogels," Journal of Bioscience and Bioengineering, vol. 106, no. 1, pp. 74-79, 2008.

[45] C. T. Jayasuriya, M. B. Goldring, R. Terek, and Q. Chen, "Matrilin-3 Induction of IL-1 receptor antagonist Is required for up-regulating collagen II and aggrecan and down-regulating ADAMTS-5 gene expression," Arthritis Research \& Therapy, vol. 14, no. 5, article R197, 2012.

[46] J. B. Vincourt, S. Etienne, L. Grossin et al., "Matrilin-3 switches from anti- to pro-anabolic upon integration to the extracellular matrix," Matrix Biology, vol. 31, no. 5, pp. 290-298, 2012.

[47] J. Erenpreisa and H. I. Roach, "Epigenetic selection as a possible component of transdifferentiation. Further study of the commitment of hypertrophic chondrocytes to become osteocytes," Mechanisms of Ageing and Development, vol. 87, no. 3, pp. 165182, 1996.

[48] R. M. Borzi, I. Mazzetti, G. Magagnoli et al., "Growth-related oncogene $\alpha$ induction of apoptosis in osteoarthritis chondrocytes," Arthritis and Rheumatism, vol. 46, no. 12, pp. 3201-3211, 2002.

[49] C. Tavera, T. Abribat, P. Reboul et al., "IGF and IGF-binding protein system in the synovial fluid of osteoarthritic and rheumatoid arthritic patients," Osteoarthritis and Cartilage, vol. 4, no. 4, pp. 263-274, 1996.

[50] Y. Du, Y. Wang, L. Wang et al., "Cartilage oligomeric matrix protein inhibits vascular smooth muscle calcification by interacting with bone morphogenetic protein-2," Circulation Research, vol. 108, no. 8, pp. 917-928, 2011.
[51] S. C. K. M. Motaung, P. E. Di Cesare, and A. Hari Reddi, "Differential response of cartilage oligomeric matrix protein (COMP) to morphogens of bone morphogenetic protein/transforming growth factor- $\beta$ family in the surface, middle and deep zones of articular cartilage," Journal of Tissue Engineering and Regenerative Medicine, vol. 5, no. 6, pp. e87-e96, 2011.

[52] C. M. Thomas, R. Murray, and M. Sharif, "Chondrocyte apoptosis determined by caspase- 3 expression varies with fibronectin distribution in equine articular cartilage," International Journal of Rheumatic Diseases, vol. 14, no. 3, pp. 290-297, 2011.

[53] J. Hecht, E. Hayes, M. Snuggs et al., "Calreticulin, PDI, Grp94 and $\mathrm{BiP}$ chaperone proteins are associated with retained COMP in pseudoachondroplasia chondrocytes," Matrix Biology, vol. 20, no. 4, pp. 251-262, 2001.

[54] J. Vranka, A. Mokashi, D. R. Keene et al., "Selective intracellular retention of extracellular matrix proteins and chaperones associated with pseudoachondroplasia," Matrix Biology, vol. 20, no. 7, pp. 439-450, 2001.

[55] R. Dinser, F. Zaucke, F. Kreppe et al., "Pseudoachondroplasia is caused through both intra- and extracellular pathogenic pathways," Journal of Clinical Investigation, vol. 110, no. 4, pp. 505-513, 2002.

[56] T.-L. L. Chen, J. W. Stevens, W. G. Cole, J. T. Hecht, and B. M. Vertel, "Cell-type specific trafficking of expressed mutant COMP in a cell culture model for PSACH," Matrix Biology, vol. 23, no. 7, pp. 433-444, 2004.

[57] J. T. Hecht and E. H. Sage, "Retention of the matricellular protein SPARC in the endoplasmic reticulum of chondrocytes from patients with pseudoachondroplasia," Journal of Histochemistry and Cytochemistry, vol. 54, no. 3, pp. 269-274, 2006.

[58] K. L. Posey and J. T. Hecht, "The role of cartilage oligomeric matrix protein (COMP) in skeletal disease," Current Drug Targets, vol. 9, no. 10, pp. 869-877, 2008.

[59] K. L. Posey, A. C. Veerisetty, P. Liu et al., "An inducible cartilage oligomeric matrix protein mouse model recapitulates human pseudoachondroplasia phenotype," American Journal of Pathology, vol. 175, no. 4, pp. 1555-1563, 2009.

[60] S. A. Abbah, W. W. Lu, S. L. Peng et al., "Extracellular matrix stability of primary mammalian chondrocytes and intervertebral disc cells cultured in alginate-based microbead hydrogels," Cell Transplantation, vol. 17, no. 10-11, pp. 1181-1192, 2008.

[61] L. G. Alexopoulos, L. A. Setton, and F. Guilak, "The biomechanical role of the chondrocyte pericellular matrix in articular cartilage," Acta Biomaterialia, vol. 1, no. 3, pp. 317-325, 2005.

[62] L. G. Alexopoulos, G. M. Williams, M. L. Upton, L. A. Setton, and F. Guilak, "Osteoarthritic changes in the biphasic mechanical properties of the chondrocyte pericellular matrix in articular cartilage," Journal of Biomechanics, vol. 38, no. 3, pp. 509-517, 2005.

[63] E. Kim, F. Guilak, and M. A. Haider, "The dynamic mechanical environment of the chondrocyte: a biphasic finite element model of cell-matrix interactions under cyclic compressive loading," Journal of Biomechanical Engineering, vol. 130, no. 6, Article ID 061009, 2008.

[64] E. Kim, F. Guilak, and M. A. Haider, "An axisymmetric boundary element model for determination of articular cartilage pericellular matrix properties in situ via inverse analysis of chondron deformation," Journal of Biomechanical Engineering, vol. 132, no. 3, Article ID 031011, 2010. 
[65] M. Wong, M. Siegrist, and X. Cao, "Cyclic compression of articular cartilage explants is associated with progressive consolidation and altered expression pattern of extracellular matrix proteins," Matrix Biology, vol. 18, no. 4, pp. 391-399, 1999.

[66] A. L. Stevens, J. S. Wishnok, D. H. Chai, A. J. Grodzinsky, and S. R. Tannenbaum, "A sodium dodecyl sulfate-polyacrylamide gel electrophoresis-liquid chromatography tandem mass spectrometry analysis of bovine cartilage tissue response to mechanical compression injury and the inflammatory cytokines tumor necrosis factor $\alpha$ and interleukin-1 $\beta$," Arthritis and Rheumatism, vol. 58, no. 2, pp. 489-500, 2008. 


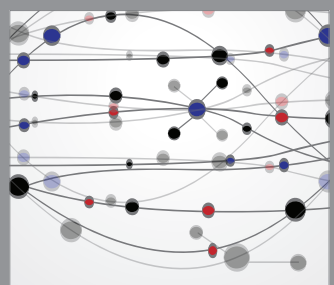

The Scientific World Journal
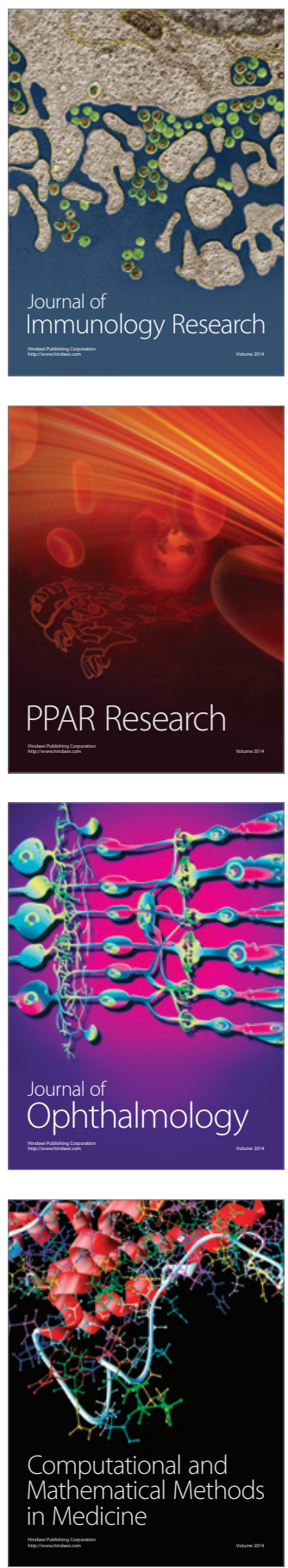

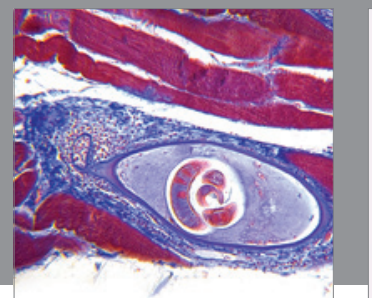

Gastroenterology

Research and Practice
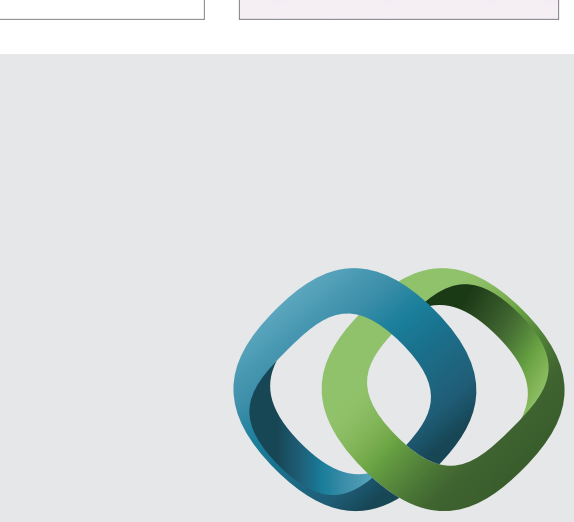

\section{Hindawi}

Submit your manuscripts at

http://www.hindawi.com
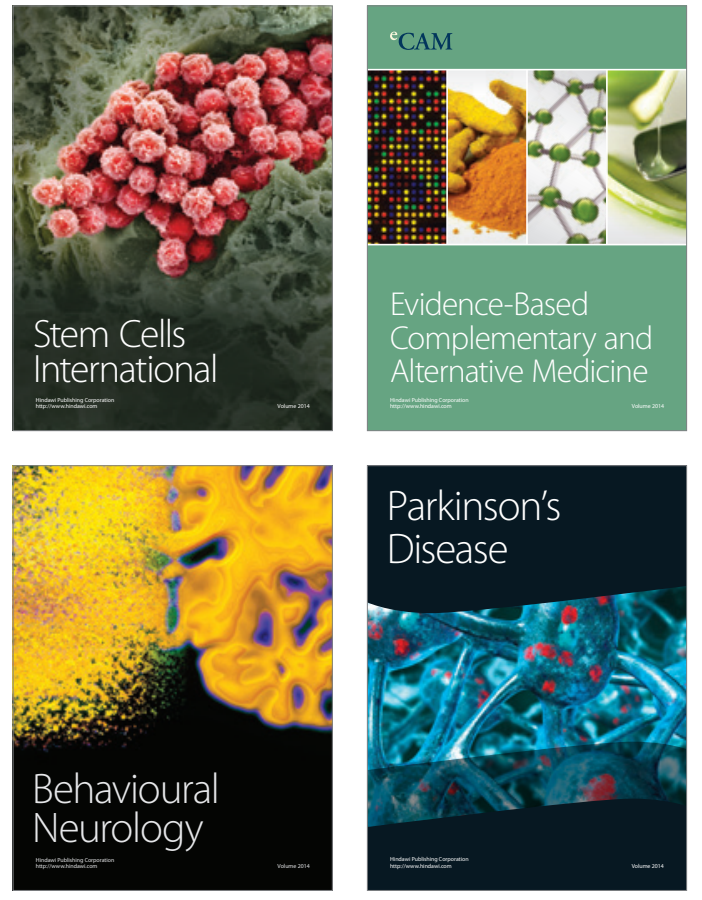
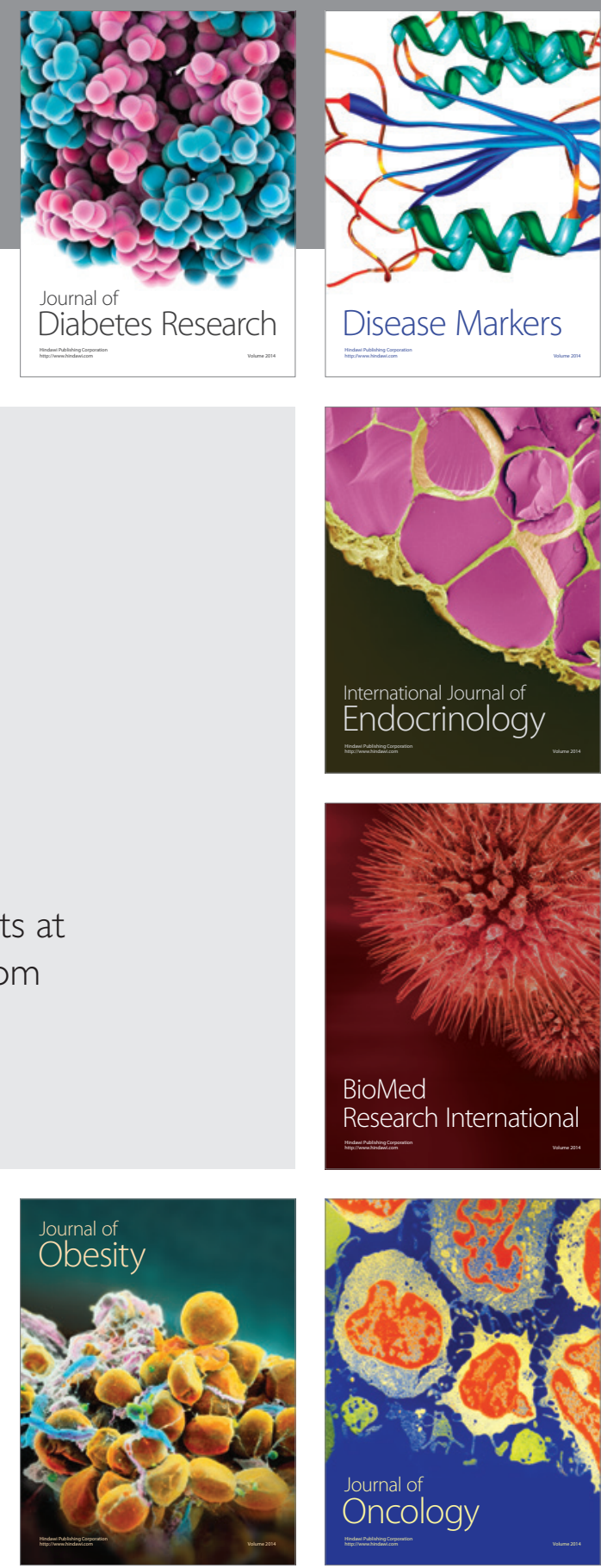

Disease Markers
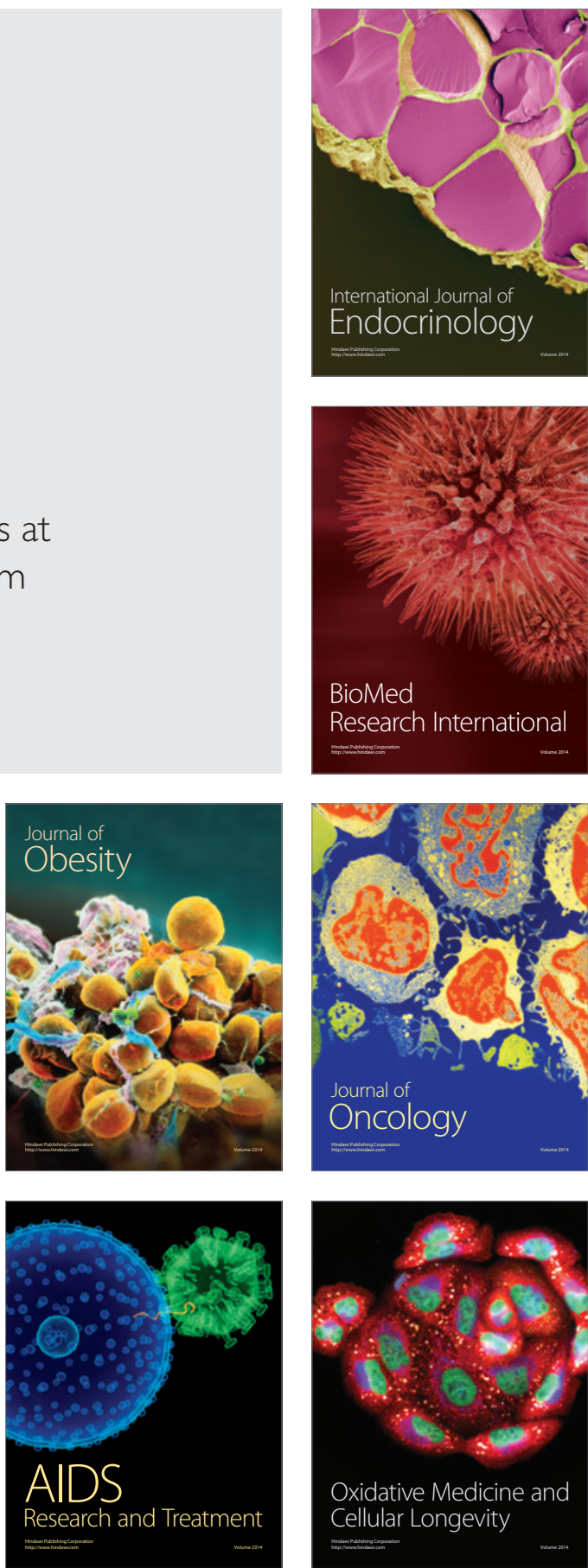